\title{
Parameter Estimation of Spring-Damping System using Unconstrained Optimization by the Quasi-Newton Methods using Line Search Techniques
}

\author{
Namala Dheeraj Kumar $^{1 *}$, V. Surendranath ${ }^{2}$ \\ ${ }^{1}$ Department of Aerospace, SRM University \\ ${ }^{2}$ Department of Aerospace, IISC Bengaluru
}

\author{
*Corresponding Author email: \\ dheerajkumar837@gmail.com \\ Article History \\ Received: 28 April 2018 \\ Revised: 03 July 2018 \\ Accepted: 10 August 2018 \\ Published: 09 September 2018 \\ Student(s) \\ - Namala Dheeraj Kumar

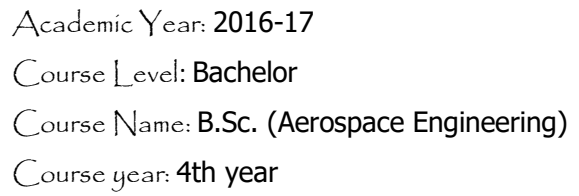

\section{Introduction}

Optimization, also known as mathematical programming, A collection of mathematical principles and methods used for solving quantitative problems in many disciplines, including physics, engineering mainly aerospace as discussed in many reports to find out the parameters of the aircraft. The subject grew from a realization that quantitative problems in manifestly different disciplines have important mathematical elements in common. Because of this commonality, many problems can be formulated and solved by using the unified set of ideas and methods that make up the field of optimization. Quasi-Newton methods are methods used to either find zeroes or local maxima and minima of functions, as an alternative to Newton's method. They can be used if the Jacobian or Hessian is unavailable or is too expensive to compute at every iteration. This is used to minimize the cost function by maximum likelihood method. The method of maximum likelihood corresponds to many well-known estimation methods. Output error has matured into one of the most widely accepted and used methods in the aerospace industry for estimating unknown model

Copyright (C) 2018. The Author(s). Published by AIJR Publisher.

This is an open access article under Creative Commons Attribution-NonCommercial 4.0 International (CC BY-NC 4.0) license, which permits any non-commercial use, distribution, adaptation, and reproduction in any medium, as long as the original work is 
parameters from measured flight data. It is a straightforward method based on maximum likelihood estimation that uses realistic assumptions consistent with standard flight test practices and the real physical situation, and also possesses useful analytic properties as discussed in many reports [1],[2],[3],[4],[5] In this paper the above methodology is used for a spring-damping system to study the system. Many systems exhibit oscillatory behavior when they are disturbed from their position of static equilibrium. A mass suspended from a spring, for example, might, if pulled and released, bounce up and down. On each bounce, the system is trying to return to its equilibrium position but overshoots it. Sometimes losses (e.g. frictional) damp the system and can cause the oscillations to gradually decay in amplitude towards zero or attenuate. This system can be represented by second order differential equation. This paper presents a method to estimate parameters of spring damping system using a optimization technique. These methods are being widely used most importantly the mass-spring damping system, in Amplitude Frequency Characteristics [6], estimation of contact parameters [6], Vibration Isolation [7]. A similar work was done in 2018 using algebraic identification method [8]. This paper deals with quasi newton approach.

\section{Methodology}

Let us consider a mass ' $m$ ' attached to the spring, when displaced from the mean position it oscillates about the mean position as the time progress the amplitude attenuates due to damping this system is represented by the below equation (1).

$$
m \ddot{x}+c \dot{x}+k x=0
$$

Where

$\mathrm{m}$ is the mass of the body.

$\mathrm{c}$ is the damping coefficient.

$\mathrm{k}$ is the spring constant.

The above equation is solved by using O.D. E (ordinary differential equation) solver of Matlab. The resulting equation produced after solving gives the variation of displacement from the mean position with the time. This displacement equation (2) is the function of time ( $\mathrm{t})$, spring constant $(\mathrm{k})$ and damping coefficient $(\mathrm{c})$.

$x(t)=f(k, c, m, t)$

The equation (1) is differentiated with respect to parameters and these to partial differential equations (3),

(4) are given below.

$$
\begin{aligned}
& \frac{d x(t)}{d k}=f(k, c, m, t) \\
& \frac{d x(t)}{d c}=f(k, c, m, t)
\end{aligned}
$$

The Quasi-newton direction is given by the below equation (5).

$$
d=-b * g(x)
$$

where

$\mathrm{g}(\mathrm{x})=$ gradient of the function

$\mathrm{b}=$ inverse of hessian matrix

$x_{k+1}=x_{k}+a * d$

Where a is step length

The Davidon-Fletcher-Powell formula for

Is given by the following equation (7).

$$
B_{k+1}=\left(I-y_{k} * \Delta x_{k}^{T} / y_{k}^{T} * \Delta x_{k}\right) * B_{k} *\left(I-\Delta x_{k} * y_{k}^{T} / y_{k}^{T} * \Delta x_{k}\right)+\frac{y_{k} * y_{k}^{T}}{y_{k}^{T} * \Delta x_{k}}--(7)
$$


The other method Broyden-Fletcher-Goldfarb-Shanno formula for updation is given by the below equation (8) and change is step is given (9) and gradient change is given by (10)

$B_{k+1}=B_{k}+\frac{y_{k} * y_{k}^{T}}{y_{k}^{T} * \Delta x_{k}}-\frac{B_{k} \Delta x_{k}\left(B_{k} \Delta x_{k}\right)^{T}}{\Delta x_{k}{ }^{T} B_{k} \Delta x_{k}} \ldots$

Where

$$
\begin{gathered}
\Delta x_{k}=x_{k+1}-x_{k} \\
y_{k}=\mathrm{g}\left(x_{k+1}\right)-\mathrm{g}\left(x_{k}\right)-(9)
\end{gathered}
$$

The backtracking line search is a line search method to determine the maximum amount to move along a given search direction. It involves starting with a relatively large estimate of the step size for movement along the search direction, and iteratively shrinking the step size until a decrease of the objective function is observed that adequately corresponds to the decrease that is expected, based on the local gradient of the objective function. The Armijo's Goldstein condition is used to give addition bounds for the step change which helps to converge the optimization problem the rule which governs this condition is given below (11).

$a=r_{o} * a$

where

$r_{o}=$ multiplication factor to change steplength

Armijo's condition is given by (12), (13)

$\Phi_{1}(a)=\mathrm{f}(x)+c_{1} \mathrm{a} g^{T} \mathrm{~d}$

$f(x+a d) \leq \Phi_{1}(a)$

Goldstein condition is given by (14),(15)

$$
\begin{aligned}
& \Phi_{2}(a)=\mathrm{f}(x)+c_{2} \mathrm{a} g^{T} \mathrm{~d} \\
& f(x+a d) \geq \Phi_{2}(a) .
\end{aligned}
$$

The maximum likelihood estimator minimizes the below cost function (16)

$J(\varepsilon)=0.5 * \sum_{i=1}^{N}\left[x\left(t_{i}\right)-x_{\varepsilon}\left(t_{i}\right)\right]^{2}$

Where

$$
\begin{aligned}
& x_{\varepsilon}=\text { computed value } \\
& \mathrm{x}=\text { measured value }
\end{aligned}
$$

The gradient of the cost function is given by the following equation (17).

$$
g(J(\varepsilon))=-\sum_{i=1}^{N}\left[x\left(t_{i}\right)-x_{\varepsilon}\left(t_{i}\right)\right] *\left[g\left(x_{\varepsilon}\left(t_{i}\right)\right)\right]
$$

\section{Experimentation}

To obtain the experimental displacement data of spring damping system, experiment is done in the laboratory with a mass attached to the spring and one end of the spring is fixed to the rigid support which is free to oscillate about the equilibrium position when given any disturbance. The displacement sensor which is connected to a data acquisition system is used to track the information in terms of voltage, the calibration of the sensor is done, and the sensitivity of the sensor is measured. This voltage is converted to the distance with the help of sensitivity of the sensor. The entire experimental process is carried for 10 seconds and the initial displacement of $2 \mathrm{~cm}$ is given the corresponding data is acquired by the data acquisition system as shown in Figure 1 variation of Displacement with time. The setup is well maintained without any external disturbance.

Experimental values of

Spring constant $(\mathrm{k})=3 \mathrm{~N} / \mathrm{m}$

Damping coefficient $(\mathrm{c})=0.5 \mathrm{~N} / \mathrm{ms}$

Mass of the $\operatorname{body}(\mathrm{m})=2 \mathrm{Kg}$ 


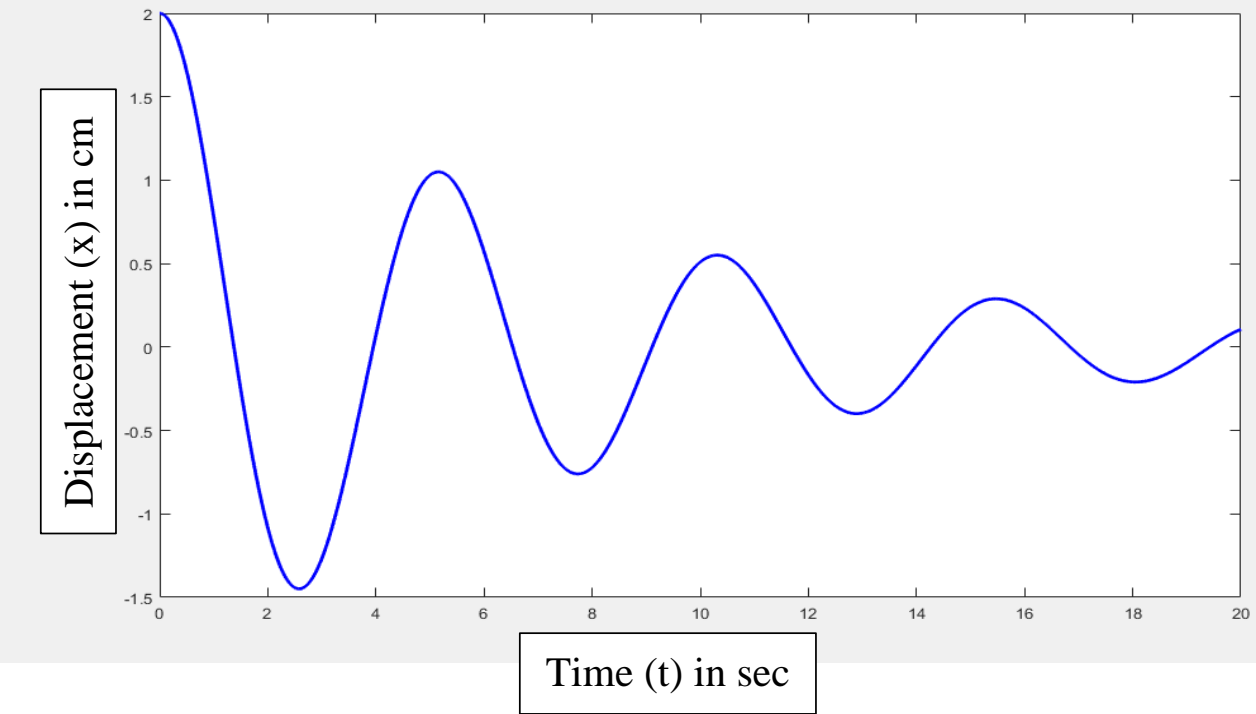

Figure 1: Shows variation of Displacement with time

\section{$4 \quad$ Results}

The results obtained by the above methodology after a number of iterations for two methods with same initial guess are given below. The blue graph in the figure indicates the experimental data as shown in figure 2 to 4 . And the red graph indicated the computed displacement data of the body attached to the spring after the nth iteration. This method is used to estimate the parameters of spring damping system accurately as Algebraic Identification Method [9].

\subsection{Broyden-Fletcher-Goldfarb-Shanno Method}

The problem is converged after 38 iterations by using Broyden-Fletcher-Goldfarb-Shanno method .in the final iteration both the graphs overlapped. Figure 2,3,4 shows variation of Displacement with time of Iteration-1,32,38. The computed values of

Spring constant $(\mathrm{k})=2.9999997 \mathrm{~N} / \mathrm{m}$

Damping coefficient $(\mathrm{c})=0.50000036 \mathrm{~N} / \mathrm{ms}$

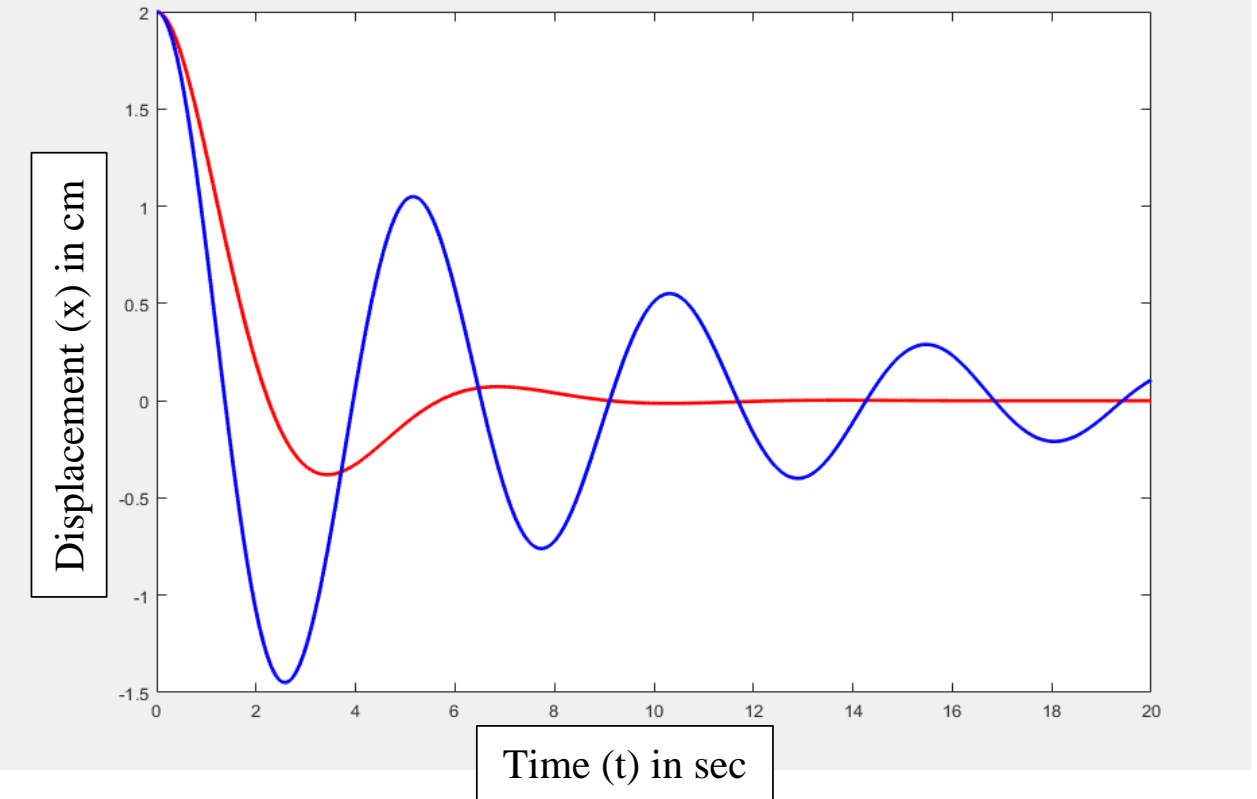

Figure 2: Shows variation of Displacement with time of Iteration-1 


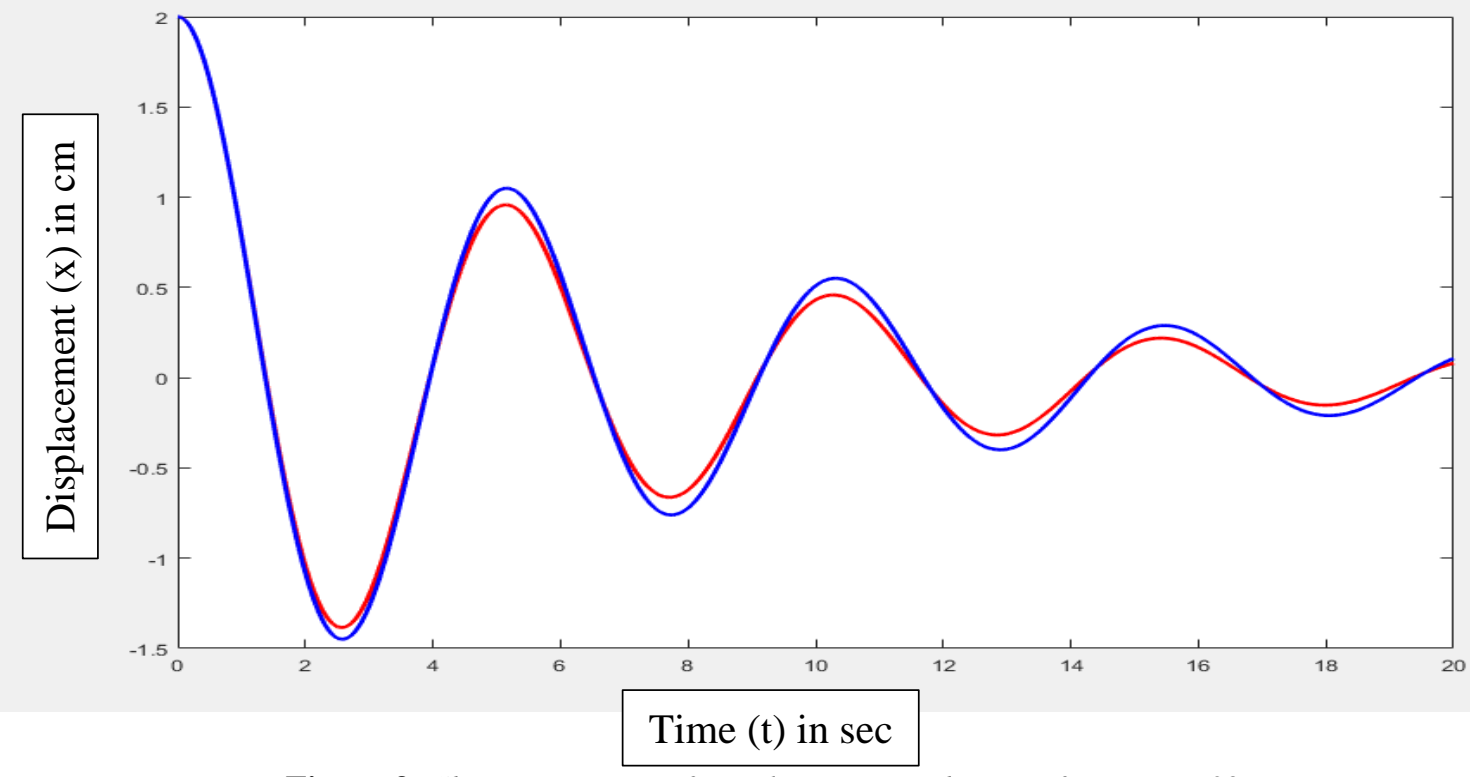

Figure 3: Shows variation of Displacement with time of Iteration-32

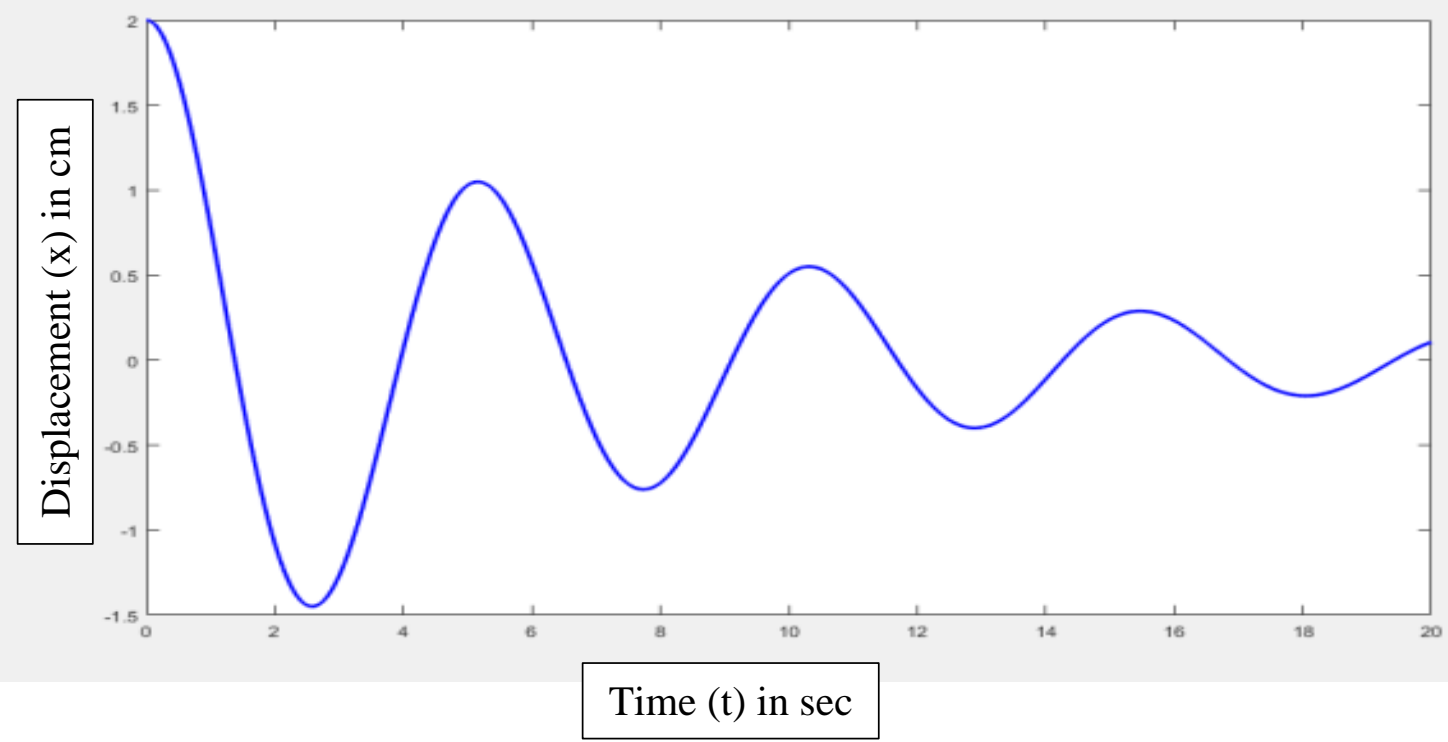

Figure 4: Shows variation of Displacement with time of Iteration-38

\subsection{Davidon-Fletcher-Powell Method}

The problem is converged after 38 iterations by using davidon-Fletcher-Powell method. In the final iteration both the graphs overlapped as shown in figure 7 and figure 5 to 6 represent the graph at iterations 62 and 145. The computed values of

Spring constant $(\mathrm{k})=2.9999966 \mathrm{~N} / \mathrm{m}$

Damping coefficient $(\mathrm{c})=0.4999982 \mathrm{~N} / \mathrm{ms}$ 


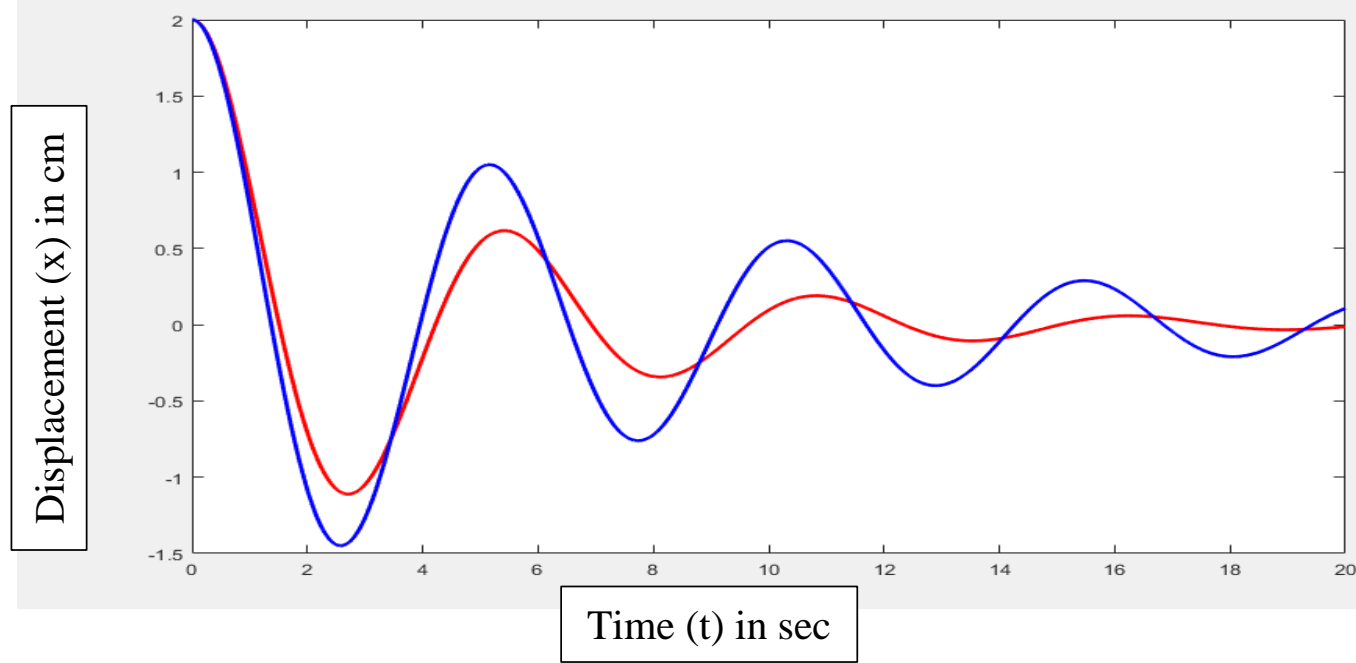

Figure 5: Shows variation of Displacement with time of Iteration-62

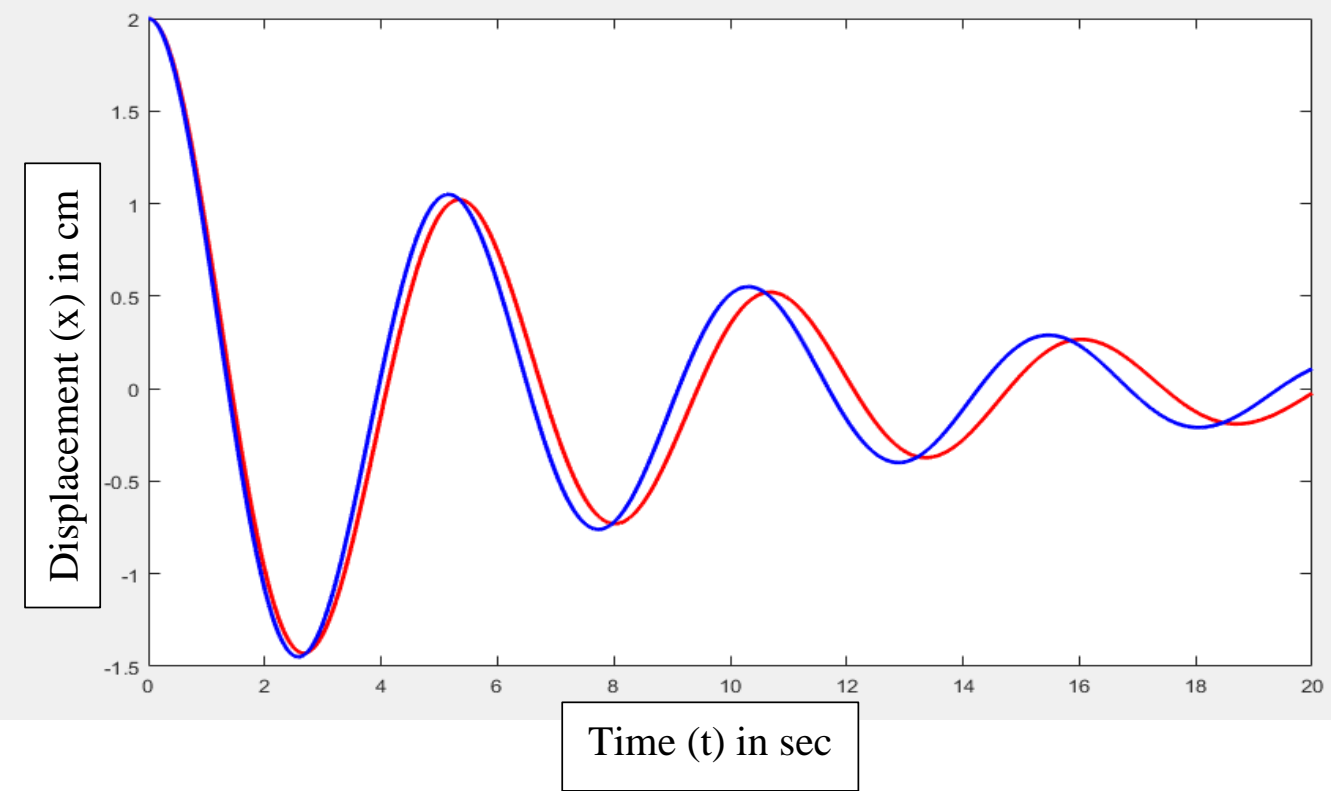

Figure 6: Shows variation of Displacement with time of Iteration-145

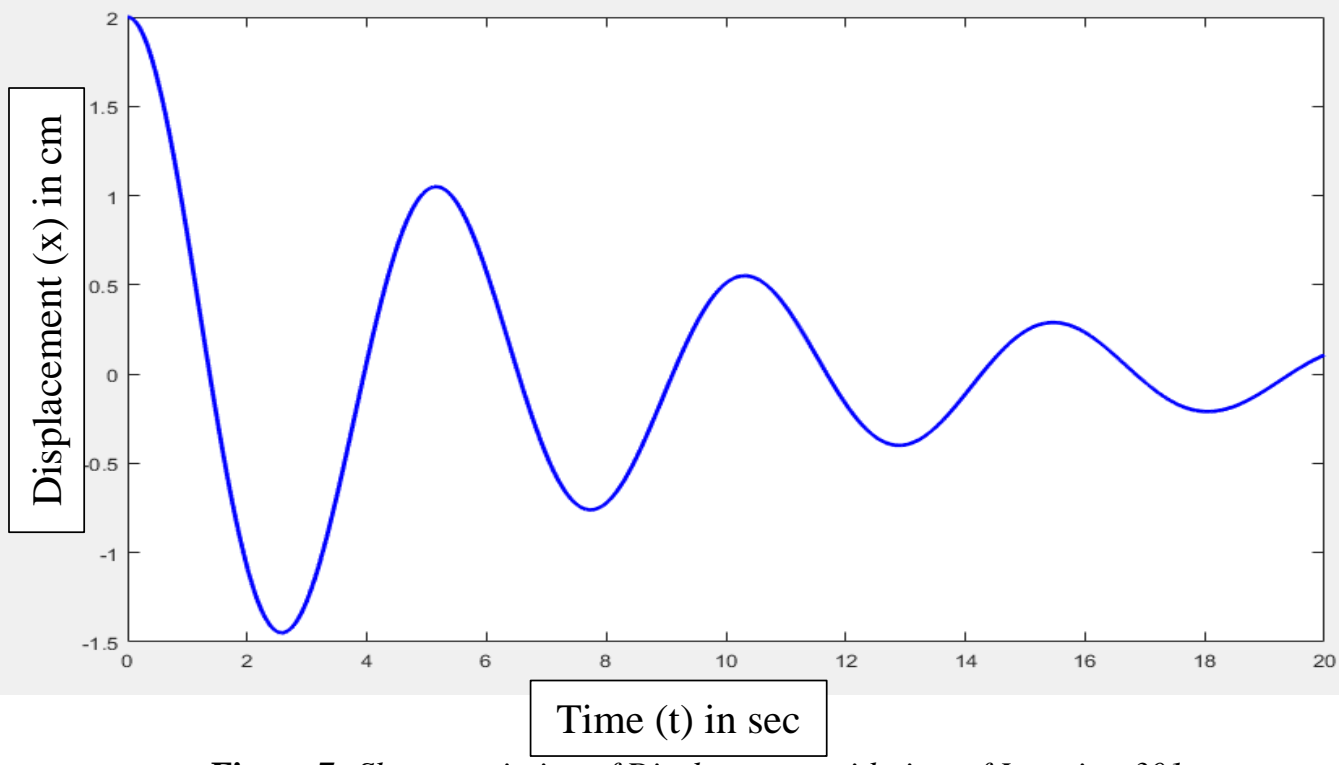

Figure 7: Shows variation of Displacement with time of Iteration-301 


\section{Conclusion}

The Quasi newton approach estimation for mass, damping and stiffness parameters for mechanical system of spring mass system using acceleration measurements has been proposed for the above results obtained by two quasi-newton methods (B.F.G.S and Davidson-Fletcher-Powell) It can be depicted that the former method converges in few iterations and robust when compared to the later one. The percentage of error with the experimental procedure is insignificant. Figure 8 shows variation of Displacement with time of Davidson-Fletcher-Powell and Broyden-Fletcher-Goldfarb-Shanno. However, these methods sensitive to the initial guess. So the quasi newton methods can be used to study the different systems by understanding the behaviour of the system. Future studies will include the application of these quasi newton identification approach to the on-line parametric estimation problem of nonlinear vibrating mechanical systems.

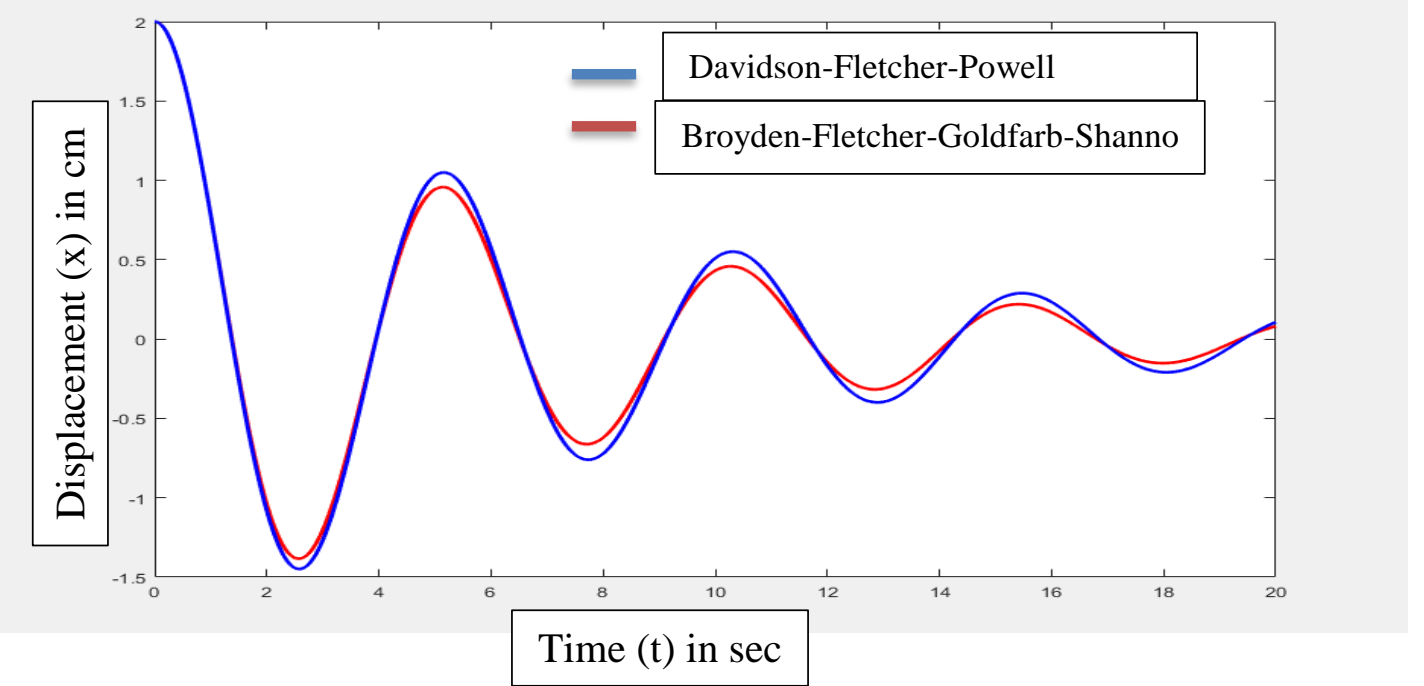

Figure 8: Shows variation of Displacement with time of Davidson-Fletcher-Powell and Broyden-Fletcher-Goldfarb-Shanno

\section{How to Cite this Article:}

D. Namala and V. Surendranath, "Parameter Estimation of Spring-Damping System using Unconstrained Optimization by the Quasi-Newton Methods using Line Search Techniques", Adv. J. Grad. Res., vol. 5, no. 1, pp. 1-7, Sep. 2018. doi: https://doi.org/10.21467/ajgr.5.1.1-7

\section{References}

[1] J. Becedas., G. Mamani., V. Feliu-Batlle and H. Sira-Ramírez, Algebraic Identification Method for Mass-Spring-Damper System, Proceedings of the World Congress on Engineering and Computer Science 2007.

[2] Alessandri, A., Baglietto, M., and Battistelli, G. (2008). Moving-horizon state estimation for nonlinear discretetime systems: New stability results and approximation schemes. Automatica, 44(7), 1753-1765.

[3] B. A. Lloyd and S. Kirac and G. Székely and M. Harders, Identification of Dynamic Mass Spring Parameters for Deformable Body Simulation, EUROGRAPHICS 2008.

[4] Mark Nagurka, Richard Marklin. Measurement of Stiffness and Damping Characteristics of Computer Keyboard Keys, Journal of Dynamic Systems, Measurement, and Control, 2005.

[5] J. Becedas, G. Mamani, V. Feliu-Batlle and H. Sira-Ramírez, Algebraic Identification Method for Mass-Spring-Damper System, Proceedings of the World Congress on Engineering and Computer Science 2007.

[6] O. Kohl, Identification of Parameters of Stiffness and Damping Based on the Amplitude Frequency Characteristics, Springer International Publishing Switzerland 2016.

[7] L. Pesik, A. Skarolek, O. Kohl, Vibration Isolation Pneumatic System with a Throttle Valve, Springer International Publishing Switzerland 2016.

[8] Francisco Beltrán Carbajal, Gerardo Silva Navarro and Luis Gerardo Trujillo Franco, ONLINE PARAMETRIC IDENTIFICATION OF MASS-SPRING-DAMPER MECHANICAL SYSTEMS USING ACCELERATION MEASUREMENTS, Pistas Educativas, No. 128 (SENIE 2017), febrero 2018.

Publish your research article in AIJR journals-

Online Submission and Tracking | Peer-Reviewed | Rapid decision | Immediate Publication after acceptance | Articles freely available online | Retain full copyright of your article.

Submit your article at journals.aijr.in 